\title{
Possible nonequilibrium imprint in the cosmic background at low frequencies
}

\author{
Marco Baiesi $\odot,{ }^{1,2}$ Carlo Burigana $\odot,{ }^{3,4,5}$ Livia Conti $\odot,{ }^{2, *}$ Gianmaria Falasco, ${ }^{6}$ Christian Maes $\odot,{ }^{7}$ \\ Lamberto Rondoni $\odot{ }^{8,9}$ and Tiziana Trombetti ${ }^{3,10}$ \\ ${ }^{1}$ Dipartimento di Fisica e Astronomia, Università degli Studi di Padova, 35121 Padova, Italy \\ ${ }^{2}$ Sezione di Padova, Istituto Nazionale di Fisica Nucleare, 35131 Padova, Italy \\ ${ }^{3}$ Istituto di Radioastronomia, Istituto Nazionale di Astrofisica, 40129 Bologna, Italy \\ ${ }^{4}$ Dipartimento di Fisica e Scienze della Terra, Università di Ferrara, 44122 Ferrara, Italy \\ ${ }^{5}$ Sezione di Bologna, Istituto Nazionale di Fisica Nucleare, 40126 Bologna, Italy \\ ${ }^{6}$ Complex Systems and Statistical Mechanics, Physics and Materials Science Research Unit, \\ University of Luxembourg, 1511 Luxembourg, Luxembourg \\ ${ }^{7}$ Instituut voor Theoretische Fysica, KU Leuven, 3001 Leuven, Belgium \\ ${ }^{8}$ Dipartimento di Scienze Matematiche, Politecnico di Torino, 10129 Torino, Italy \\ ${ }^{9}$ Sezione di Torino, Istituto Nazionale di Fisica Nucleare, 10125 Torino, Italy \\ ${ }^{10}$ Istituto di Scienze Marine, Consiglio Nazionale delle Ricerche, 40129 Bologna, Italy
}

(Received 26 August 2019; accepted 3 February 2020; published 26 February 2020)

\begin{abstract}
Cosmic background radiation has been observed to deviate from the Planck law expected from a blackbody at $\sim 2.7 \mathrm{~K}$ at frequencies below $\sim 3 \mathrm{GHz}$. We discuss the abundance of the low-energy photons from the perspective of nonequilibrium statistical mechanics. We propose a mechanism of stochastic frequency diffusion, the counterpart to stochastic acceleration for charged particles in a turbulent plasma, to modify the standard Kompaneets equation. The resulting violation of the Einstein relation allows us to take advantage of low-frequency localization and finally leads to photon cooling. The modified equation predicts a frequency distribution in agreement with the absolute temperature measurements of the cosmic background radiation down to about $20 \mathrm{MHz}$, for which we offer an updated compilation. In that sense, the so-called space roar we observe today is interpreted as a nonequilibrium echo of the early universe and more specifically of nonequilibrium conditions in the primordial plasma.
\end{abstract}

DOI: 10.1103/PhysRevResearch.2.013210

\section{INTRODUCTION}

The cosmic microwave background $(\mathrm{CMB})$ is a prime witness to the physics of the early universe. According to the standard model of physical cosmology, it carries information about an epoch before neutral atoms were formed, dating from some $10^{5}$ years after the Big Bang (see [1,2] for reviews). It is generally assumed then that matter and radiation were approximately in thermodynamic equilibrium, owing to the high efficiency of Compton scattering, bremsstrahlung, and radiative Compton processes, with characteristic timescales much shorter than the cosmic expansion timescale. At the recombination era, when electrons and protons formed hydrogen atoms, light decoupled from matter and the photons started to move almost freely through the expanding universe, influenced by secondary effects only, such as the cosmological reionization associated with the formation of early stars and galaxies and the weak gravitational lensing induced by cosmic

\footnotetext{
*livia.conti@pd.infn.it

Published by the American Physical Society under the terms of the Creative Commons Attribution 4.0 International license. Further distribution of this work must maintain attribution to the author(s) and the published article's title, journal citation, and DOI.
}

structures (see [3,4] for reviews and [5,6] for recent analyses). As a result, the distribution function of the $\mathrm{CMB}$ photons at later times $t$ is supposed to follow the blackbody spectrum at an equilibrium temperature $T(t)$. After a delicate subtraction of the intervening astrophysical emissions, the cosmic background appears today very close to a blackbody radiation [7] at a temperature of about $2.7 \mathrm{~K}$, peaking at about $160 \mathrm{GHz}$, in very good agreement with the Planck spectrum from about $10 \mathrm{GHz}$ up to about $600 \mathrm{GHz}$.

However, there is evidence of a systematic deviation from the Planck law of a blackbody at about $2.7 \mathrm{~K}$ at low frequencies, in the radio tail of the cosmic background. That aspect was brought to attention by two independent types of observations: the CMB absolute temperature excess measured by the second generation absolute radiometer for cosmology, astrophysics, and diffuse emission instrument (ARCADE 2) [8] and the anomalously strong absorption of the redshifted $21-\mathrm{cm}$ line from neutral hydrogen measured by the experiment to detect the global epoch of reionization signature (EDGES) [9]. After consideration of possible instrumental errors and after subtracting galactic and extragalactic sources of low-frequency radiation, a strong residual emission remains in ARCADE 2 data [10], which is much larger than predicted by the standard theory of CMB spectral distortions. Consistently, the intriguing EDGES absorption profile amplitude, about 2-3 times larger than expected, could be explained 
by a much stronger background radiation with respect to standard predictions (see [11] for an alternative explanation assuming that the primordial hydrogen gas was much colder than expected). The scientific literature in fact abounds with experimental data from low-frequency radio surveys, some going back a long time: After subtraction for galactic and extragalactic contributions, they all show an excess of soft, i.e., low-frequency, photons. A concise description of the cosmic background spectrum data considered in this work is given in Sec. II.

We repeat that the CMB spectrum theory assumes (near-) equilibrium conditions, e.g., up to the time of recombination. The equilibrium distribution, the Planck law, is the quantum analog of the Maxwell distribution for a classical ideal gas and as emphasized already by the pioneers of statistical mechanics, it is the distribution to be typically expected as a consequence of counting with Bose statistics. For the kinetics and relaxation to the Planck distribution, we recall in Sec. III the Kompaneets equation [12], which is used in that context. It describes the evolution of the photon spectrum due to repeated Compton scattering off a thermal bath of nonrelativistic electrons, possibly towards the equilibrium Planck distribution. In the Kompaneets equation, the Planck law entails zero current (in frequency space) as a result of the detailed balance between diffusion and drift. That arises from the analog of the Einstein relation or the second fluctuation-dissipation relation as it is called in the (classical) Fokker-Planck equation. However, kinetically, there is localization at low frequencies, as implied by the proportionality $v^{2}$ of the kinetic coefficients: The number (or density) of soft photons does not change easily as the interaction with the plasma is damped at low frequency. It is that kinetic aspect that is crucially important when (even slightly) violating the Einstein relation.

In this paper we no longer assume that the universe at $t \simeq 1 \mathrm{~s}$ after the Big Bang was in thermodynamic equilibrium for the relevant degrees of freedom. Instead, in Sec. IV, we propose an additional turbulent diffusion in frequency space. Its origin is the assumed chaotic nature of the turbulent plasma, where stochastic acceleration is caused by space-time random force fields [13-15]. Quite independent of the detailed mechanism, the central-limit theorem gives an extra contribution to the diffusivity proportional to $v^{-1}$ (best fit $v^{-1.3}$ ) to be added to the standard diffusive contribution proportional to $v^{2}$, which has its origin in phase-space calculations. For sufficiently low frequencies (actually, within the megahertz to gigahertz range), the $v^{-1}$ obviously dominates and leads to photon cooling. The behavior for even much lower frequencies is unexplored today (see also Appendix B).

Finally, in Sec. V, we check whether the suggested nonequilibrium change in the Kompaneets equation allows one to reproduce the main features of the observational data, in particular the observed excess in the cosmic background at low frequency. Good agreement is remarkably easy to obtain. The imbalance between drift and diffusion in the Kompaneets equation, resulting in what is here an effective pumping towards low frequency, is thus understood as a nonequilibrium feature.

While the argument is statistical, it is based on the presence of nonequilibrium dynamical activity in the primordial plasma. The suggested mechanism is formally similar to the one for population selection in various nonequilibrium distributions, as has been discussed, for example, for population inversion in lasers [16], for kinetic proofreading in protein synthesis [17], and for suprathermal kappa-distributions in space plasma [18]. It may be theoretically summarized in the so-called blowtorch theorem [19,20]: Here, violating the Einstein relation and adding a low-frequency diffusion immediately leads to the abundance of soft photons.

Nonequilibrium effects in the early universe have not been discussed extensively so far and details on the precise mechanism cannot be provided at this point. So far, the origin of nonequilibrium features can only be thought to reside ultimately with gravitational degrees of freedom that have influenced the nature of light and matter, as may be expected in strongly non-Newtonian regimes of gravity. Furthermore, the supposed presence of frequency diffusion most likely leads to much slower relaxation.

The hypothesis that the low-frequency excess in the CMB is a nonequilibrium imprint, originating at (or ultimately before) the time of the primordial plasma, does not stand alone though. We mentioned already the blowtorch theorem and its relevance to population inversion. We have also been led to investigate such an idea by the various analogies we see with the phenomenon of low-frequency spectral power enhancement that has been observed in a number of different nonequilibrium systems, including disordered systems [21,22], fluids [23,24], driven macromolecules [25], and vibrating solids [26]. We also refer to the theoretical models in $[27,28]$ for a different type of population inversion.

\section{OBSERVATIONAL FRAMEWORK}

Measurements of the absolute temperature of the cosmic background have been performed since the CMB discovery by Penzias and Wilson [29] at $4.08 \mathrm{GHz}$. In this paper we consider the cosmic background absolute temperature data on the basis of the available measurements, including their quoted global errors that are related to limited experimental sensitivity, residual systematic effects, observed sky areas, and uncertainties in foreground signal subtraction. We provide an updated and almost complete data compilation that we use in the comparison with the predicted photon density. This is necessary for evaluating our theoretical model of Sec. V. In order to make our analysis essentially independent of specific data selections, we typically avoid using particular combinations of subsets of data, a possibility that we consider just for some comparisons (see also Appendix A); an exhaustive investigation of the implications of adopting the various possible subsets of data is beyond the scope of the present study. In particular, we exploit the following.

(i) We use the data listed in Table 1 of the ARCADE 2 data interpretation paper [10], but not the far-infrared absolute spectrophotometer (FIRAS) "condensed" data at $250 \mathrm{GHz}$.

(ii) We avail ourselves of the data compilation from the various experiments reported in Table 1 of [30], where joint constraints on early and late CMB spectral distortions were presented. 
(iii) We make use of the measurements by the TRIS experiment together with the long-wavelength compilation in Table 1 reported in [31].

(iv) We benefit from the extremely accurate measurements by FIRAS on board the Cosmic Background Explorer (COBE) [7,32]. We take from [7] the measurements at the five lowest FIRAS frequencies, while the results in [32] are used above $68 \mathrm{GHz}$. A little rescaling is applied to the FIRAS data to account for the last absolute temperature calibration by Fixsen [33] at $T^{*}=2.72548 \mathrm{~K}$. We do not include the data by the COBRA experiment and by the analysis of molecular lines, as they fall in the same range of the much more accurate FIRAS measurements.

(v) We employ the recent data between 0.04 and $0.08 \mathrm{GHz}$ from Dowell and Taylor [34]. They refer to the extragalactic signal without any subtraction of the known contribution by extragalactic sources, which we perform as described below. Note that the value adopted by the authors for the extragalactic background temperature at $408 \mathrm{MHz}$ is consistent with the one in Table 1 from [10], but not with the value in the subset of the older data in Table 1 of [30].

As is well known, excluding the ARCADE 2 measurements, the averaged temperature of the data at $1 \mathrm{GHz} \lesssim v \lesssim$ $30 \mathrm{GHz}$ is slightly below the FIRAS temperature determination at $v \gtrsim 30 \mathrm{GHz}$. On the other hand, the measurements below $\sim 1 \mathrm{GHz}$ and the excess at $\simeq 3.3 \mathrm{GHz}$ claimed by ARCADE 2 indicate a remarkable temperature increase in the radio tail of the background radiation.

One should consider possible necessary corrections to the data, as sources other than CMB may have contributed. The relevance of the accurate subtraction clearly emerges in the ARCADE 2 data about the residual extragalactic emission presented in Table 1 of [10]. The authors derive the extragalactic signal after the subtraction of the galactic emission. Their residual extragalactic emission assumes the model by Gervasi et al. [35] to describe the global contribution by unresolved extragalactic radio sources, expressed in terms of the antenna temperature $T_{\text {ant }}(v)=\left(c^{2} / 2 v^{2} k_{\mathrm{B}}\right) \int_{S_{\min }}^{S_{\max }} S N^{\prime}(v, S) d S$. Here $c$, $k_{\mathrm{B}}, \nu, S$, and $N^{\prime}(v, S)$ are the light speed, the Boltzmann constant, the photon frequency, the source flux density, and the source differential number counts, respectively. However, recent studies [36-39] suggest an increase of $N^{\prime}(\nu, S)$ up to a factor $\sim 3$ at $\sim 10 \mu \mathrm{Jy}$ and a factor of $\sim 1.5$ at $\sim 100 \mu \mathrm{Jy}$ with respect to the differential number counts in [35], likely to be ascribed to faint star forming galaxies and radio-quiet active galactic nuclei. By simply rescaling at faint fluxes the differential number counts in [35] by such factors, we find a larger contribution of unresolved extragalactic radio sources (of about $30 \%$, when expressed in terms of antenna temperature, since only a fraction of the global contribution by unresolved extragalactic radio sources comes from sources at faint flux densities), always to be subtracted from the signal to derive the residual extragalactic emission.

The data we adopt in this study for the cosmic background absolute temperature are listed in Appendix A (see Table I). We report the background temperatures according to quoted papers, by assuming the signal treatment originally performed by authors (second column). For the data where the model in [35] was applied to subtract the global contribution by unresolved extragalactic radio sources such as, for instance, in [10], we report also the background temperature derived by applying the higher subtraction described above to account for possible higher differential number counts at faint flux densities (third column). For the case of the data in [34] we perform the subtraction using both the recipe in [35] and this higher model. See also Appendix A for further details. The data in Table I are displayed in Fig. 1; Fig. 1(a) refers to the background temperatures in the second column and Fig. 1(b) to the ones in the third column. When compared to the quoted uncertainties, the higher subtraction, translated in equivalent thermodynamic temperature (see Table I), gives appreciable changes between 0.022 and $0.08 \mathrm{GHz}$ for the two TRIS measurements around $0.7 \mathrm{GHz}$ and, but only weakly, for the two ARCADE 2 measurements around $3.3 \mathrm{GHz}$.

Many explanations have been tried to account for the residual low-frequency excess and we cannot mention all attempts. For example, the diffuse free-free emission associated with cosmological reionization has been considered as one way to explain the ARCADE 2 and the radio background excess, but the signal spectral shape is steeper than that predicted for the free-free distortion [10]. Furthermore, the signal amplitude is much larger than those derived for a broad set of models (see [40,41]). Efforts have also been dedicated to explain the low-frequency background signal excess and the EDGES absorption profile in terms of astrophysical emissions, possibly in combination with particle physics phenomena (see [11,42-46]).

So far, there is no agreement in explaining the intriguing and still even questioned data (see [47-50]). The present study is aimed at taking a very different route than previous studies, to consider the background radiation excess in the radio tail as a true cosmological signal and to explain it in terms of nonequilibrium statistical mechanics.

\section{KOMPANEETS EQUATION NEAR EQUILIBRIUM}

The fundamental equation describing the kinetics of Compton scattering of photons and thermal electrons, which is relevant for the relaxation to the Planck distribution in the primordial plasma as well, was introduced by Kompaneets [12] and by Weymann [51]. It is assumed that the energy exchanges are nonrelativistic at electron temperature $T_{e}$ with $k_{B} T_{e} \ll m_{e} c^{2}$ and for photon energies $h v \ll m_{e} c^{2}$. Then the dimensionless occupation number $n(t, v)$ at time $t$ and frequency $v$, obtained from the spectral energy density $E_{v}$ of the radiation via $n=\left(c^{3} / 8 \pi h v^{3}\right) E_{v}$, is shown to satisfy

$$
\partial_{t} n=\frac{\sigma_{T} N_{e} h}{m_{e} c} \frac{1}{v^{2}} \partial_{\nu}\left\{v^{4}\left[\frac{k_{\mathrm{B}} T_{e}}{h} \partial_{\nu} n+(1+n) n\right]\right\},
$$

where $\sigma_{T}$ is the Thomson cross section and $N_{e}$ is the electron density. We refer to the literature [52,53] for details of the derivation of (1). The starting point is a Boltzmann equation for photons interacting with a plasma where the main mechanism is elastic Compton scattering between electrons and photons. This is thought to be the primary mechanism for the (partial) thermalization of the CMB.

We use a rescaled version of that Kompaneets equation

$$
\partial_{\tau} n=\frac{1}{v^{2}} \partial_{\nu}\left\{v^{4}\left[\frac{k_{\mathrm{B}} T_{e}}{h} \partial_{\nu} n+(1+n) n\right]\right\}
$$


for the time evolution of the photon occupation number $n(\tau, v)$ with rescaled time $\tau=h t \sigma_{T} N_{e} / m_{e} c$, which is irrelevant for the stationary solution we are after. The stationary solution of (2) for which the expression between square brackets vanishes, $k_{\mathrm{B}} T_{e} \partial_{\nu} n_{\mathrm{eq}}+h\left(1+n_{\mathrm{eq}}\right) n_{\mathrm{eq}}=0$, is the equilibrium Bose-Einstein distribution

$$
n_{\mathrm{eq}}(v)=\frac{1}{e^{h v /\left(k_{\mathrm{B}} T_{e}\right)+C}-1},
$$

which reduces to the Planck law for integration constant $C=$ 0 (photon chemical potential). Assuming thermal equilibrium between electrons and photons, with the usual, proportional to $1+z$, temperature scaling for redshift $z$ due to cosmic expansion, we have $T_{e}(z)=T^{*}(1+z)$, which is the same scaling as for the photon frequency.

In terms of the photon density (per unit frequency) defined as $\rho(\tau, v):=v^{2} n(\tau, v)$, with the prefactor $v^{2}$ being proportional to the density of states, Eq. (2) reads

$$
\partial_{\tau} \rho=\partial_{\nu}\left[\frac{k_{\mathrm{B}} T_{e}}{h} v^{2} \partial_{\nu} \rho+v\left(v-2 \frac{k_{\mathrm{B}} T_{e}}{h}\right) \rho+\rho^{2}\right] .
$$

It is the nonlinear term $\sim n^{2}$ in (2) that makes the "lowfrequency" Rayleigh-Jeans contribution $n_{\mathrm{RJ}}(v) \sim v^{-1}$ or, in (4),

$$
\rho_{\mathrm{RJ}}=\frac{k_{B} T_{e}}{h} v
$$

for the Rayleigh-Jeans density corresponding to (4). Without that nonlinearity the stationary solution would be the Wien spectrum $n_{\text {Wien }}(v) \propto \exp \left(-h v / k_{\mathrm{B}} T_{e}\right)$, which is a good approximation for high frequencies. We emphasize that in all events the Planck law solves (2) because it balances the diffusion term (second-order derivative) with the drift term (first-order derivative), independent of the prefactor $v^{4}$ in front of the square bracket. That is the usual scenario for detailed balance (or reversible) dynamics [54], for which the stationary solution shows zero current in the frequency domain.

The next important observation is the emergence of a localization effect at low frequencies, realized by the power $v^{4}$ in (2). Dynamically the escape rates away from low frequency are strongly damped, which implies, for example, slower relaxation for initial conditions peaking at low frequencies. That frequency dependence can be read off from the KleinNishina cross section (for Thomson to Compton scattering). Again, that kinetics is not visible in the equilibrium Planck distribution but it does play a role dynamically. In fact, the low-frequency localization is a typical wave phenomenon: Scattering is limited at low frequencies/large wavelengths.

To be complete we note that, in the above, we considered the Kompaneets equation including only Compton scattering. The evolution equation for the photon occupation number could be described by a "generalized" Kompaneets equation accounting also for other physical processes in the plasma and coupled to an evolution equation for the electron temperature [55]. Unavoidable photon production/absorption processes operating in cosmic plasma [2] include double (or radiative) Compton scattering [56-58], bremsstrahlung [59,60], and in the presence of primordial magnetic fields the cyclotron process [61]. In (near)equilibrium conditions their rates are derived assuming again detailed balance and, consequently, in combination with the Compton scattering, they tend to reestablish a Planckian spectrum, as the reversible (zero-current) stationary solution. Other photon production/absorption processes are predicted in exotic models. Heating and cooling mechanisms not directly originating photon production/absorption can be also effectively added as source terms in the Kompaneets equation or in the evolution equation of the electron temperature, according to a variety of almost standard or exotic processes. The resulting spectra mainly depend, at high redshifts, on the process epoch, the global amount of injected photon energy and number density, and the overall energy exchange and at low redshifts also on the details of the considered mechanism (see [62]).

In the following sections we neglect the effects of such additional mechanisms, focusing instead on the implication of "violating" the Einstein relation in the (simplest and most elementary version of the) Kompaneets equation. One must realize that the relaxation times are probably largely affected by the additional turbulent diffusion, especially in the lowfrequency regime. That can be inferred from the effective (modified) Kompaneets equation, without specifying the detailed physics.

\section{TURBULENT ACCELERATION IN FREQUENCY SPACE}

In what follows we consider the analog of stochastic acceleration, but in frequency space, as a possible additional source of momentum transfer to photons. Stochastic acceleration is the net acceleration that particles may be expected to experience when moving under the influence of randomlyspace-time-varying force fields [13-15]. It is closely related to turbulent diffusion whereby additional diffusion in velocity space is generated due to random or chaotic accelerations. As the net (spatially or temporally averaged) force is zero, the effect is a cumulative result on the level of most significant fluctuations, as expressed by the central-limit theorem. As an alternative picture, we can imagine particles in a nonequilibrium medium, for which the weak coupling or the Van Hove limit $[63,64]$ produces an additional diffusive contribution in their effective Fokker-Planck description. In the equilibrium case, noise is accompanied with friction in an amount prescribed by the fluctuation-dissipation relation (see also the next section). In the case of stochastic acceleration, the source of noise tends to be macroscopic nonequilibrium with no or almost no compensation in terms of friction. Without a thermal background, stochastic acceleration leads to runaway solutions as noted, for example, by Fermi in his explanation of the origin of cosmic (high-energy) radiation [65]. Such an effect, that is, turbulent diffusion on top of thermal processes, is well known in plasmas and has been described since the discovery of Taylor dispersion [66]. Stochastic acceleration in frequency space originates from high-frequency cancellation of energy transfer. As an example for coupled oscillators, we refer to [67-69], where rotors with high energy tend to decouple from their neighbors due to fast oscillation of the forces. In general, the variance of the total momentum exchange scales like the inverse of the square root of the energy, as the following argument shows. 
Additionally to the thermal effects in the standard Kompaneets equation (2), we consider energy transfers (over a small time interval $\sim \epsilon$ ) that relate momenta $m v_{i}+h v_{i} / c \leftrightarrow$ $h v_{\epsilon} / c+m v_{\epsilon}$ over quasirandom nonequilibrium forcing. To be specific, from the point of view of the charged particles, we suppose the presence of a random nonconservative force field $F$, e.g., generated by electromagnetic wave turbulence. Let us suppose a classical picture where the force $F_{S}(x)$ changes in time $s$ at frequency $v_{i}$ while varying in space $x$ over a length $\ell$. For an electron moving at high velocity $v$ through the random medium, the incurred force $G_{s}$ as a function of time $s$ thus decorrelates $^{1}$ at a rate $\tau^{-1}$, which scales like $\tau^{-1} \propto v_{i}+\ell^{-1} v$. The total momentum exchange over an arbitrarily small time $\epsilon$ is the time integral of that force $G_{s}$, which means that the energy given to the photon is

$$
h v_{\epsilon}-h v_{i}=c \int_{0}^{\epsilon} d s G_{s}=c \int_{0}^{\epsilon} d s \tilde{G}_{s / \tau}=c \tau \int_{0}^{\epsilon / \tau} d u \tilde{G}_{u},
$$

where $\tilde{G}_{u}$ is the rescaled force field, which now has persistence time of order one. We assume that the force field is random with zero average and sufficiently ergodic. Then, for small persistence time $\tau$, the central-limit theorem ${ }^{2}$ applies to the last integral and

$$
h v_{\epsilon}-h v_{i}=c \tau \sqrt{\frac{\epsilon}{\tau}} Z=c \sqrt{\tau \epsilon} Z
$$

where $Z$ is a zero-mean Gaussian with finite variance containing more details about the forcing. In particular, from (7) and with $\tau^{-1}$ growing proportionally to $v_{i}$, we expect a variance

$$
\left\langle\left|h v_{\epsilon}-h v_{i}\right|^{2}\right\rangle \propto \frac{1}{v_{i}} \epsilon
$$

as a function of the initial photon frequency. In summary, by the oscillations in the electromagnetic field, time in momentum exchange gets measured in units of $1 / v$. As a result, small frequencies are more affected by the process. An alternative argument similar to the momentum transfer formula (6) is obtained from inspecting time averages of oscillating integrals.

Equation (8) yields an additional diffusion in frequency space as the photon energy over a small time $\epsilon$ scales with $\epsilon$ and with a diffusivity which is proportional to the inverse frequency. That provides an extra diffusion to add to the Kompaneets equation, as from the Fokker-Planck theorem (see [70]). As a consequence of (8), we conclude by suggesting an addition to the Kompaneets equation (2) for $\partial_{\tau} n$ [see also (10) and the discussion at the beginning of Sec. V] proportional to

$$
\frac{1}{v^{2}} \partial_{v}\left\{v^{2} \frac{1}{v} \partial_{v} n\right\}
$$

\footnotetext{
${ }^{1}$ In the sense of a persistence time for the external noise experienced by the electron.

${ }^{2}$ If $X_{i}$ are sufficiently independent with zero mean $\lim _{n \rightarrow \infty} \frac{1}{n}$ $\sum_{i=1}^{n} X_{i}=0$ and finite variance $\lim _{n \rightarrow \infty} \frac{1}{n} \sum_{i=1}^{n} X_{i}^{2}=\sigma^{2}$, then as $n \rightarrow \infty, \sum_{i=1}^{n} X_{i}=\sqrt{n} Z$ in the distribution, where $Z$ is a Gaussian random variable with mean zero and variance $\sigma^{2}$.
}

The $1 / v$ is directly from (8), while the factors $v^{2}$ originate from the three-dimensional Laplacian in reciprocal space with the wave vector $k$ having amplitude $|k|=v / c$. We therefore write now

$$
\begin{aligned}
\partial_{\tau} n=\frac{1}{v^{2}} \partial_{\nu}\left\{v^{4}\left[\frac{k_{\mathrm{B}} T_{e}}{h} \partial_{\nu} n+(1+n) n\right]\right\} \\
+\frac{1}{v^{2}} \partial_{\nu}\left\{v^{2} \frac{k_{\mathrm{B}} T_{e}}{h} B(v) \partial_{\nu} n\right\},
\end{aligned}
$$

with

$$
B(v) \propto \frac{1}{v} \text { for } v \gg v_{1}
$$

The first term on the right-hand side of (10) is the (original) thermal contribution (2), while the second term is purely diffusive and breaks the balance that before led to the BoseEinstein equilibrium distribution (3): When indeed the dimensionless factor $B(v) / v^{2}$ depends on $v$, we cannot interpret (10) as a reversible Kompaneets equation (2) with a new (effectively global) temperature.

In (11) we also indicate a frequency regime $v \gg v_{1}$ for the proposed $B(v) \propto 1 / v$. A precise quantitative value for $v_{1}$, such that the above applies for $v \gg v_{1}$, is difficult to determine from the above heuristics, but the analysis of the data (in the next section, to be summarized in Figs. 1 and 2) suggests $v_{1}<10^{-2} \mathrm{GHz}$.

The above argument is general and powerful as it does not depend on the specific mechanisms of scattering or interaction. It is a statistical argument based on the assumption of a sufficiently chaotic energy transfer, somewhat similar to the famous Stosszahlansatz in the kinetic theory of gases. Note, however, that a nonequilibrium input is needed: The randomness is on the level of the force $F$ in (6) and the force is not allowed to be conservative (gradient of a potential) as a time-extensive integral (or path-dependent work) is necessary for the application of the central-limit theorem in (6).

The random force field $F$ appearing in the argument above is reminiscent of the intrinsic fluctuations in the CMB that were detected by COBE. Their origin is obviously an open problem for fundamental physics. However, from a general perspective, the presence of nonequilibrium dynamical activity is perhaps not surprising at all. Under the low-entropy assumption for the very early universe [71], it is not strange to believe that the primordial plasma was active and not starting in global thermal equilibrium. A far-from-equilibrium initial plasma would have very large relaxation times for the low frequencies. Analogously, in space plasmas we see suprathermal tails in the electron velocity distribution, stemming from a high-energy localization in the electronic degrees of freedom coupled to a turbulent electromagnetic field [18]. That may have contributed to the abundance of soft photons and together prevented thermalization before the radiation became free CMB and a near-steady occupation [see Eq. (15) below] was installed. The low-frequency localization, already present in the reversible Kompaneets equation, is then the final complement to the high-energy localization in the electron momentum transfer.

Obviously, details of the mechanism will need to be added, and other scenarios may be imagined. Here we just note that 

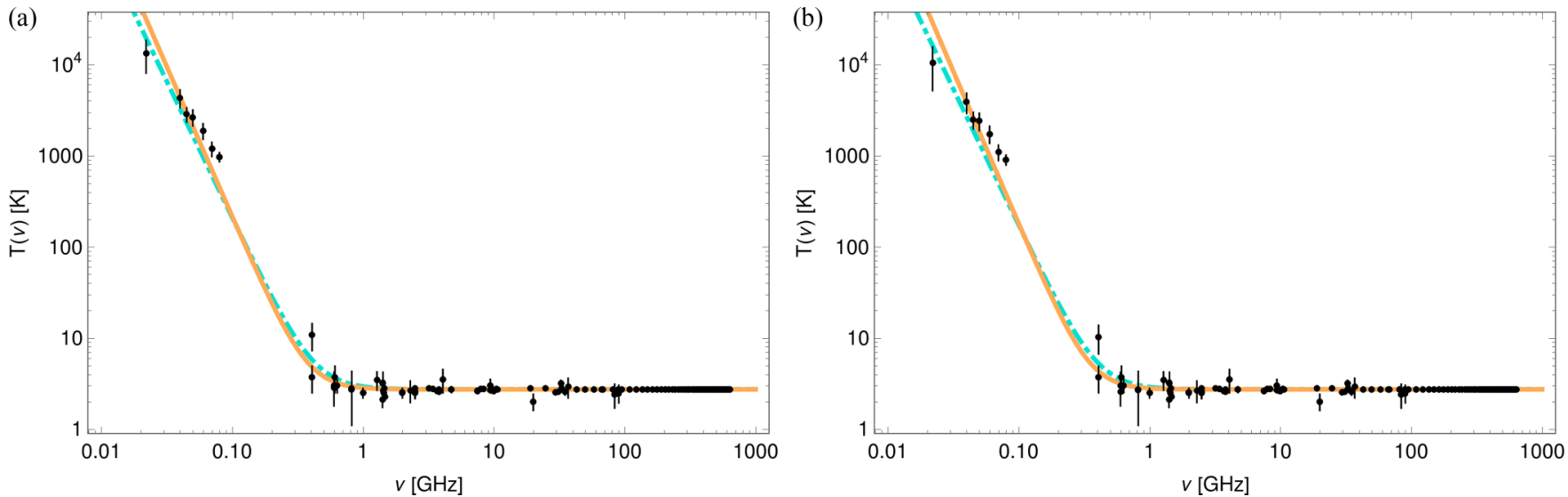

FIG. 1. Cosmic background absolute temperature as a function of the frequency (in gigahertz). The experimental data (black dots with $1 \sigma$ error bars) refer to the measurements discussed in Sec. II: (a) smaller subtraction of extragalactic signal and (b) higher subtraction (see Table I). For each panel, the turquoise dot-dashed line is the best fit of the data with $T(v)$ obtained from (17), with $\alpha=3$ and $v_{0}$ as the only free parameter: In (a) we find $v_{0}=0.42 \pm 0.04 \mathrm{GHz}$ with a reduced $\chi^{2}=2.1$, while in (b) we find $v_{0}=0.40 \pm 0.05 \mathrm{GHz}$ with a reduced $\chi^{2}=2.16$ (errors at $95 \%$ confidence level). The solid orange lines are the best fits with both $\alpha$ and $v_{0}$ as free parameters: At $95 \%$ confidence level, the fitting procedure results in (a) $\nu_{0}=0.38 \pm 0.05 \mathrm{GHz}$ and $\alpha=3.30 \pm 0.22$ with a reduced $\chi^{2}=1.91$ and (b) $\nu_{0}=0.35 \pm 0.06 \mathrm{GHz}$ and $\alpha=3.36 \pm 0.28$ with a reduced $\chi^{2}=1.93$.

a proper quantum mechanical treatment of the interactions between photons and nonequilibrium collective plasma excitations leads in the semiclassical limit [see Eq. (A9) in [72]] to the dissipative Kompaneets equation (10). Also, we refer to $[73,74]$ for other examples and derivation of low-frequency distortions due to the induced Compton scattering. Another way to transfer energy from photons to electrons is to think of pair production from high-energy photons. Pair production in a rapidly expanding universe, such as under early inflation, will then create real long-lived high-energy electrons while depleting the high-frequency photon spectrum.

\section{BREAKING THE EINSTEIN RELATION}

The present section discusses the consequences of the structure (10) and (11) that we have argued for above. At this moment it is instructive to consider an even larger class of Kompaneets equations. The modified Kompaneets equation (10) is a special case of the type

$$
\partial_{\tau} n=\frac{1}{v^{2}} \partial_{v}\left\{v^{2}\left[D(v) \partial_{v} n+\gamma(v)(1+n) n\right]\right\} .
$$

The notation suggests that $D(v)$ be thought of as a frequencydependent diffusion and $\gamma(v)$ as a frequency-dependent friction. In (2) the diffusivity $D(v)=k_{\mathrm{B}} T_{e} v^{2} / h$ and the friction coefficient $\gamma(v)=v^{2}$ are linked by the Einstein relation $D(v) / \gamma(v)=k_{\mathrm{B}} T_{e} / h$. This last property, with $D(v) / \gamma(v)$ independent of $v$, ensures the reversible solution (3). The appearance of $v^{2}$ in the diffusivity (12) is an entropic effect related to the evaluation of phase-space integrals in the derivation of the Kompaneets equation. It refers to the degeneracy of the energy for a given frequency. The real issue therefore is (11), which is additive to thermal diffusion.

Comparing (10) with (2), we retain $\gamma(v)=v^{2}$ for the friction, but the diffusivity changes by the addition of $B(v)$ for which we argued the decay (11). For the sake of data analysis,
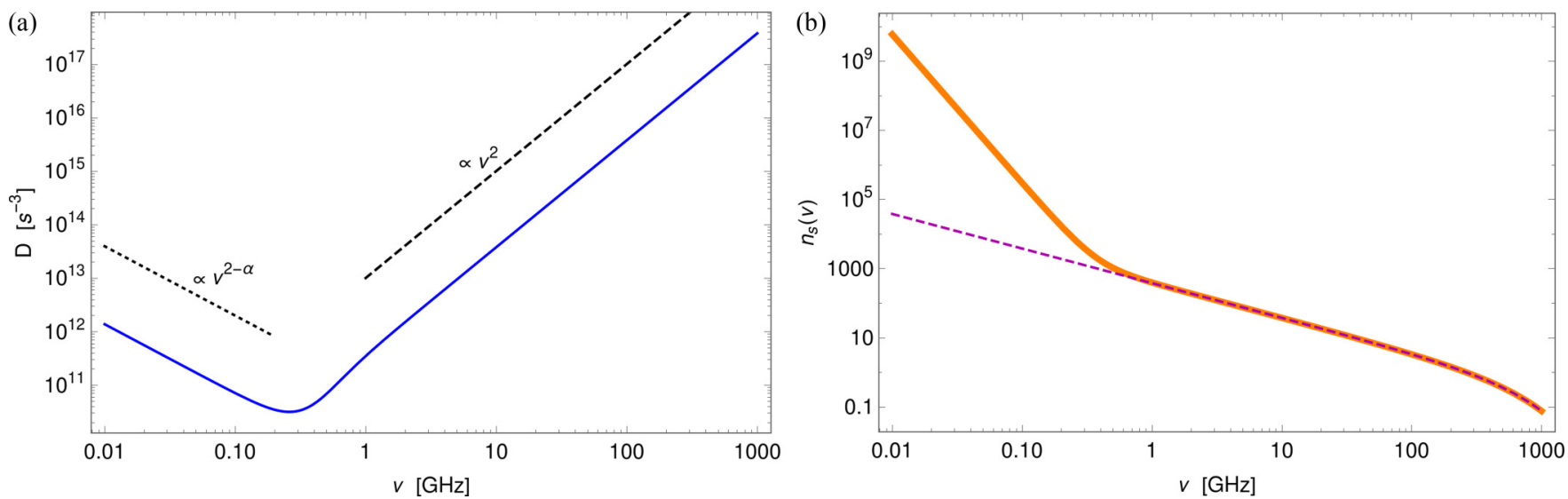

FIG. 2. For the parameters resulting from the fit of the data set in column (a) of Table I: (a) the diffusivity $D(v)$ (solid line); the dotted line represents a power law $\propto v^{2-\alpha}$ while the dashed one $\propto v^{2}$. (b) Plots of the dimensionless occupation number $n_{s}(v)$ from (15) (solid line) compared with the curve associated to a Planck law at $T^{*}$ (dashed line). 
we generalize that to the form

$$
D(v)=\frac{k_{\mathrm{B}} T_{e}}{h}\left[v^{2}+v^{2-\alpha} \frac{v_{0}^{\alpha}}{\alpha+1}\right],
$$

which corresponds to the dependence

$$
B(v)=\frac{v^{2}}{\alpha+1}\left(\frac{v}{v_{0}}\right)^{-\alpha}
$$

in (10). The argument of the preceding section gave $\alpha \simeq 3$, that is, the diffusivity changes from a behavior $D(v) \propto v^{2}$ at very large frequencies to a behavior $D(v) \propto v^{2-\alpha}$ at lower frequencies, with $\alpha \simeq 3$. The $v_{0}$ then just appears as the crossover frequency between the behavior $1 / v$ and $v^{2}$, as $v$ grows larger.

Even though the Einstein relation is violated in (10)-(14), it is quite easy to find the stationary solution of (10). This is the occupation number

$$
n_{s}(v)=\frac{1}{\left[\exp \int^{v} d v^{\prime} \frac{\gamma\left(v^{\prime}\right)}{D\left(v^{\prime}\right)}\right]-1}=\frac{1}{e^{\phi(v)}-1},
$$

where $\phi(v)=\int^{v} d v^{\prime} \gamma\left(v^{\prime}\right) / D\left(v^{\prime}\right)$ is expressed in terms of hypergeometric functions if the form (13) is assumed. However, to achieve convergence when performing fits, we replace the hypergeometric function by its approximation

$$
\phi(v):=\frac{h v}{k_{B} T_{e}} \frac{\left(v / v_{0}\right)^{\alpha}}{1+\left(v / v_{0}\right)^{\alpha}}=\frac{h v}{k_{B} T^{*}} \frac{\left(v / v_{0}\right)^{\alpha}}{1+\left(v / v_{0}\right)^{\alpha}},
$$

which has the same low-frequency and high-frequency scalings of the exact $\phi$ in (15). Since data are expressed in units of Kelvin, fits are performed with the temperature function corresponding to $\phi$,

$$
T(v)=T_{e}\left[1+\left(\frac{v}{v_{0}}\right)^{-\alpha}\right]=T^{*}\left[1+\left(\frac{v}{v_{0}}\right)^{-\alpha}\right] .
$$

In (16) and (17) and from here onward, when referring to the comparison with observational data, we are taking $T_{e}$ equal to the present CMB temperature $T^{*}$ at high frequencies.

As our first motivation for (13) and making (14) explicit, we check whether (17) fits the observations for $\alpha=3$, expected on the theoretical grounds of Sec. IV. The results of the fits are shown in Figs. 1(a) and 1(b) for the two data sets that we are considering, as detailed in Sec. II and listed in Table I of Appendix A. Only minimal differences in the retrieved best-fit parameters are obtained for the two different radio background subtractions, without relevant changes of the whole picture. Overall there is general agreement between the data and this model, with reduced $\chi^{2} \simeq 2.1$ for both data sets (see also Appendix A). We also perform a fit keeping both $v_{0}$ and $\alpha$ as free parameters; the results of the fits are also shown in Figs. 1(a) and 1(b) for the two data sets. In this case the retrieved values are $v_{0} \simeq 0.37 \mathrm{GHz}$ and $\alpha \simeq 3.3$, with a slightly lower reduced $\chi^{2} \simeq 1.9$. This exponent is significantly larger than that found using only the data in Table 1 of [10] and is likely difficult to explain in terms of synchrotron emitters. However, this exponent is close to the value $\alpha=3$ predicted in the preceding section. As anticipated, an informed breaking of the equilibrium assumption suffices to reproduce qualitatively the low-frequency excess observed in the data.
The diffusivity $D(v)$ is plotted in Fig. 2(a) for $v_{0}=$ $0.38 \mathrm{GHz}$ and $\alpha=3.3$. The effective temperature found from $k_{B} T(v):=h D(v) / \gamma(v)$ is clearly frequency dependent, $T(v) \propto\left(v_{0} / v\right)^{\alpha} T^{*}$ for small $v / v_{0}$.

The enhanced photon occupation in the low-frequency part of the spectrum is evident in Fig. 2(b), where we plot the function $n_{s}(v)$, obtained by plugging in (15) the parameters $\alpha$ and $v_{0}$ from the fits, and the equilibrium photon occupation from the Planck spectrum. We should however not take (15) as the correct behavior at ultralow frequencies (see also the discussion in Appendix B).

In general, in (12), the term proportional to $D(v) \partial_{\nu} n$ is the transfer of (undirected) energy from the electrons (the medium) to the radiation in terms of increased intensity (number of photons). The $\gamma(v)$ relates to the $v$-dependent loss of photons. The specific breaking of the Einstein relation, the last term in (10), indeed gives a noisy rate of increase of intensity with variance $B(v)$. The noise refers to the statistical origin of the additional diffusion, which is related to dynamical activity in the plasma. As discussed in Sec. IV, it is a generic effect on the level of the central-limit theorem encompassing a large number of additional energy exchanges which however on average sum to zero, i.e., do not contribute to the drift.

To further elucidate (10) we write the modification of the Kompaneets equation (4) for the density $\rho$ :

$$
\begin{aligned}
\partial_{\tau} \rho=\partial_{\nu} & \left\{\frac{k_{\mathrm{B}} T^{*}}{h}\left[v^{2}+B(v)\right] \partial_{\nu} \rho\right. \\
& \left.+\left[v^{2}-2\left(v+\frac{B(v)}{v}\right) \frac{k_{\mathrm{B}} T^{*}}{h}\right] \rho+\rho^{2}\right\} .
\end{aligned}
$$

We can now make a more rigorous analogy with the FokkerPlanck equation, as we truly deal with the photon density $\rho$ per unit frequency: In the low-frequency approximation and by substituting (14), we have

$$
\begin{aligned}
\partial_{\tau} \rho=\partial_{\nu} & \left\{\frac{k_{\mathrm{B}} T^{*}}{h} B(v) \partial_{\nu} \rho-2 \frac{B(v)}{v} \frac{k_{\mathrm{B}} T^{*}}{h} \rho+\rho^{2}\right\} \\
=\partial_{\nu} & \left\{\frac{k_{\mathrm{B}} T^{*}}{h} \frac{v^{2}}{\alpha+1}\left(\frac{v}{v_{0}}\right)^{-\alpha} \partial_{\nu} \rho\right. \\
& \left.-2 \frac{k_{\mathrm{B}} T^{*}}{h} \frac{v}{\alpha+1}\left(\frac{v}{v_{0}}\right)^{-\alpha} \rho+\rho^{2}\right\} .
\end{aligned}
$$

The (nonequilibrium) insertion of $B(v) \propto v^{2-\alpha}$ increases the diffusion constant for small frequencies, but there is also negative friction for small frequencies via the term $B(v) / v \sim$ $v^{1-\alpha}$. The amplitude of the nonlinear term proportional to $\rho^{2}$ is unchanged of order one, which reflects the essential localization as it derives from $\gamma(v)=v^{2}$. The stationary solution of (19) is the modified Rayleigh-Jeans law (low-frequency regime in nonequilibrium)

$$
\rho_{\mathrm{mRJ}}(v)=\frac{k_{B} T^{*}}{h} v\left(\frac{v}{v_{0}}\right)^{-\alpha} .
$$

As we recall that $\alpha \simeq 3$, that shows of course a drastic increase of the density with respect to the usual (low-frequency regime in equilibrium) Rayleigh-Jeans case (5) where $\rho_{\mathrm{RJ}}(v)=$ $k_{B} T^{*} v / h$. 


\section{CONCLUSION AND OUTLOOK}

While the ultimate trigger of the additional photon intensity at low frequencies is arguably to be found in the original plasma, in the epoch from the quark to the hadron age of the universe, we have not considered here essential modifications to the usual Compton scattering theory between photons and electrons or to photon interactions. Instead of searching for more subtle aspects of QED as candidates to clarify the puzzling appearance of the space roar, our arguments have been statistical and kinetic.

We have modified the Kompaneets equation within an effective nonequilibrium scenario by introducing a frequencydependent diffusion. That leads to a violation of the Einstein relation and of the balance between diffusion and friction. The result is a clear enhancement of lower photon frequencies compatible with the best data available for the cosmic background radiation. One crucial ingredient is already present in the (reversible) Kompaneets equation: the low-frequency localization. The other ingredient is stochastic acceleration in frequency space as the result of nonequilibrium dynamical activity. Here a statistical argument (central-limit theorem) applies up to and above all details on fundamental interactions. It implies an extra source of diffusion where the diffusivity is inversely proportional to the photon frequency. It is the combination of the low-frequency localization and that turbulent diffusion that creates a (new) stationary frequency distribution for the (nonequilibrium) Kompaneets equation. As a summary, the argument of stochastic frequency acceleration has yielded the modification of the Kompaneets equation

$$
\begin{aligned}
\partial_{\tau} n=\frac{1}{v^{2}} \partial_{\nu} & \left\{v^{4}\left[\frac{k_{\mathrm{B}} T^{*}}{h} \partial_{\nu} n+(1+n) n\right]\right\} \\
& +\frac{1}{v^{2}} \partial_{\nu}\left\{v^{2} \frac{k_{\mathrm{B}} T^{*}}{h} \frac{v_{0}^{\alpha}}{(\alpha+1) v^{\alpha-2}} \partial_{\nu} n\right\}
\end{aligned}
$$

to be applied in the whole frequency range $v>20 \mathrm{MHz}$ and where $v_{0}$ and $\alpha \simeq 3$ are the only fitting parameters. We have tested our theory by calculating the resulting frequencydependent (effective) temperature of the cosmic background in a very wide range, including frequencies where excess is observed, achieving reasonable agreement with the whole data set for $v_{0} \simeq 0.3-0.4 \mathrm{GHz}$ and remarkably even when setting $\alpha=3$.

What seems mandatory for future explorations is an experimental effort devoted at more precise estimates of the cosmic background in the low-frequency tail, from about $10-20 \mathrm{GHz}$ downward. The frequency region between 0.1 and $0.4 \mathrm{GHz}$ is of particular relevance because no experimental data are available. Observations at frequencies even lower than performed so far seem to be important to test or to complement our picture, since they could reveal larger deviations from the blackbody radiation or the transition to regimes (expected towards zero frequency) different from the one explored in this work. On the other hand, the background temperature increase predicted by our model is already significant, having an amplitude comparable to or larger than those produced by unavoidable mechanisms with typical parameters, at frequencies between a few gigahertz and $10-20 \mathrm{GHz}$, a region where foreground mitigation is likely less critical and extremely accurate observations with space missions are in principle feasible. Thus, verifications of our model could take advantage of the next generation of both radio facilities and CMB dedicated projects. It would also be interesting to study the isotropy of the low-frequency excess, since the present approach neglects this issue. Finally, a more accurate comprehension of galactic and extragalactic intervening astrophysical emissions is necessary.

From the present analysis we conclude that low-frequency data may be evidence for important nonequilibrium features in the early universe, when quantum and gravitational effects were strongly influenced by special (e.g., low-entropy) conditions at the time of the Big Bang.

\section{ACKNOWLEDGMENTS}

C.B. and T.T. acknowledge partial support from the INAF PRIN SKA/CTA project FORECaST and the ASI/Physics Department of the University of Roma-Tor Vergata Grant Agreement No. 2016-24-H.0 for study activities of the Italian cosmology community. T.T. acknowledges partial support from the research program RITMARE through Contract No. SP3 WP3 AZ3 U02 and the research contract SMO at CNR/ISMAR. M.B. acknowledges support from Progetto di Ricerca Dipartimentale Grant No. BIRD173122/17. L.R. acknowledges partial support from a MIUR grant from the Dipartimenti di Eccellenza. L.R. is grateful to Xiamen University for unique hospitality.

The authors of this paper have chosen to appear in alphabetical order.

\section{APPENDIX A: DATA COMPILATION AND FIT PROCEDURE}

In Table I we report the data compilation described in Sec. II. As discussed there, for the data where the model by Gervasi et al. [35] was applied to subtract the global contribution by unresolved extragalactic radio sources, we considered also a higher subtraction to account for possible higher differential number counts at faint flux densities. These two somewhat different subtractions were also applied to the radio background data by Dowell and Taylor [34]. For the other data sets we keep the original foreground treatments performed by the authors, the differences between the two above extragalactic foreground subtraction models being in any case much smaller than the quoted uncertainties.

We perform our fit first with a two-dimensional grid in $v_{0}$ and $\alpha$, to explore the dependence of the $\chi^{2}$ on parameters and to avoid a possible wrong convergence; to overcome the finite sampling of the grid method, we then use a nonlinear minimization tool, weighting data with their inverse squared error. The fit is achieved with a Levenberg-Marquardt method from initial values $v_{0}^{\text {ini }}=0.5 \mathrm{GHz}$ and $\alpha^{\text {ini }}=4$. With significantly different initial values, the convergence of the algorithm is compromised and the final result may be easily discarded basing both on visual inspection and on the results of the grid method. Fit errors are extracted from the parameters confidence interval, with a default $95 \%$ confidence level. The reduced $\chi^{2} \simeq 1.9$ we found for our model reflects the use of 
TABLE I. Adopted data compilation for the cosmic background in terms of equivalent thermodynamic (absolute) temperature. Data are collected as described in Sec. II. (a) Model by [35] to subtract the global contribution by unresolved extragalactic radio sources. (b) Model by [35] to subtract the global contribution by unresolved extragalactic radio sources, but amplified by a factor 1.3 (in terms of antenna temperature) to account for possible higher differential number counts at faint flux densities. (c) For simplicity, we report the average of the positive and negative errors (see references for asymmetric errors, where relevant).

\begin{tabular}{|c|c|c|c|c|}
\hline$v(\mathrm{GHz})$ & $\begin{array}{l}T(\mathrm{~K}) \\
\text { (a) }\end{array}$ & $\begin{array}{l}T(\mathrm{~K}) \\
\text { (b) }\end{array}$ & $\begin{array}{l}1 \sigma \text { error }(\mathrm{K}) \\
(\mathrm{c})\end{array}$ & $\begin{array}{c}\text { Reference } \\
\text { Table } 1 \text { in [10] } \\
\text { (without condensed FIRAS at } 250 \mathrm{GHz} \text { ) }\end{array}$ \\
\hline 0.022 & 13268 & 10411 & 5229 & [76] \\
\hline 0.045 & 2843 & 2477 & 512 & [77] \\
\hline 0.408 & 10.80 & 10.21 & 3.53 & [78] \\
\hline 1.42 & 3.181 & 3.167 & 0.526 & [79] \\
\hline $3.2^{\mathrm{a}}$ & 2.7770 & 2.7759 & 0.010 & ARCADE $2[8,10]$ \\
\hline $3.41^{\mathrm{a}}$ & 2.7610 & 2.7607 & 0.008 & ARCADE $2[8,10]$ \\
\hline 7.98 & 2.761 & 2.760 & 0.013 & ARCADE $2[8,10]$ \\
\hline 8.33 & 2.742 & 2.742 & 0.015 & ARCADE $2[8,10]$ \\
\hline 9.72 & 2.73 & 2.73 & 0.005 & ARCADE $2[8,10]$ \\
\hline 10.49 & 2.738 & 2.738 & 0.006 & ARCADE $2[8,10]$ \\
\hline 29.5 & 2.529 & 2.529 & 0.155 & ARCADE $2[8,10]$ \\
\hline 31 & 2.573 & 2.573 & 0.076 & ARCADE $2[8,10]$ \\
\hline \multirow[t]{2}{*}{90} & 2.706 & 2.706 & 0.019 & ARCADE $2[8,10]$ \\
\hline & & & & $\begin{array}{l}\text { compilation in Table } 1 \text { of [30] } \\
\text { (years 1965-1975) }\end{array}$ \\
\hline 0.408 & 3.7 & & 1.2 & [80] \\
\hline 0.610 & 3.7 & & 1.2 & [80] \\
\hline 0.635 & 3.0 & & 0.5 & {$[81]$} \\
\hline 1 & 2.5 & & 0.3 & [82] \\
\hline 1.42 & 3.2 & & 1.0 & [83] \\
\hline 1.44 & 2.5 & & 0.3 & [82] \\
\hline 1.45 & 2.8 & & 0.6 & [84] \\
\hline 2 & 2.5 & & 0.3 & [82] \\
\hline 2.3 & 2.66 & & 0.7 & [85] \\
\hline 4.08 & 3.5 & & 1.0 & [29] \\
\hline 9.4 & 3.0 & & 0.5 & [86] \\
\hline 9.4 & 2.69 & & 0.185 & [87] \\
\hline 19 & 2.78 & & 0.145 & [87] \\
\hline 20 & 2.0 & & 0.4 & [88] \\
\hline 32.5 & 3.16 & & 0.26 & [89] \\
\hline 35 & 2.56 & & 0.195 & [90] \\
\hline 37 & 2.9 & & 0.7 & [91] \\
\hline 83.8 & 2.4 & & 0.7 & [92] \\
\hline 90 & 2.46 & & 0.42 & [93] \\
\hline 90 & 2.61 & & 0.25 & [94] \\
\hline \multirow[t]{2}{*}{90} & 2.48 & & 0.54 & [95] \\
\hline & & & & $\begin{array}{l}\text { compilation in Table } 1 \text { of [30] } \\
\text { (years 1985-2000) }\end{array}$ \\
\hline 0.6 & 3.0 & & 1.2 & [96] \\
\hline 0.82 & 2.7 & & 1.6 & [97] \\
\hline 1.28 & 3.45 & & 0.78 & [98] \\
\hline 1.41 & 2.11 & & 0.38 & [99] \\
\hline 1.425 & 2.65 & & 0.315 & [100] \\
\hline 1.47 & 2.26 & & 0.19 & [101] \\
\hline 2 & 2.55 & & 0.14 & [102] \\
\hline 3.8 & 2.64 & & 0.07 & [103] \\
\hline 4.75 & 2.7 & & 0.07 & [104] \\
\hline 7.5 & 2.6 & & 0.07 & [105] \\
\hline 7.5 & 2.64 & & 0.06 & [106] \\
\hline 10 & 2.62 & & 0.058 & [107] \\
\hline 10.7 & 2.730 & & 0.014 & [100] \\
\hline
\end{tabular}


TABLE I. (Continued.)

\begin{tabular}{|c|c|c|c|c|}
\hline$v(\mathrm{GHz})$ & $\begin{array}{l}T(\mathrm{~K}) \\
\text { (a) }\end{array}$ & $\begin{array}{l}T(\mathrm{~K}) \\
\quad(\mathrm{b})\end{array}$ & $\begin{array}{l}1 \sigma \text { error }(\mathrm{K}) \\
(\mathrm{c})\end{array}$ & $\begin{array}{c}\text { Reference } \\
\text { compilation in Table } 1 \text { of [30] } \\
\text { (years 1985-2000) }\end{array}$ \\
\hline 24.8 & 2.783 & & 0.089 & {$[108]$} \\
\hline 33 & 2.81 & & 0.12 & [109] \\
\hline 90 & 2.60 & & 0.09 & [110] \\
\hline \multirow[t]{2}{*}{90} & 2.712 & & 0.020 & [111] \\
\hline & (a) & (b) & & TRIS [31] \\
\hline 0.60 & 2.837 & 2.581 & 0.145 & TRIS $[31]^{\mathrm{b}}$ \\
\hline 0.82 & 2.803 & 2.695 & 0.369 & TRIS $[31]^{\mathrm{b}}$ \\
\hline \multirow[t]{2}{*}{2.5} & 2.516 & 2.511 & 0.316 & TRIS $[31]^{\mathrm{b}}$ \\
\hline & & & & compilation in Table 1 of [31] \\
\hline 3.7 & 2.59 & & 0.13 & [112] \\
\hline 4.75 & 2.71 & & 0.2 & [113] \\
\hline 2.5 & 2.62 & & 0.25 & {$[114]$} \\
\hline 2.5 & 2.79 & & 0.15 & {$[115]$} \\
\hline 2.5 & 2.5 & & 0.34 & [97] \\
\hline 3.8 & 2.56 & & 0.08 & [116] \\
\hline \multirow[t]{2}{*}{3.8} & 2.71 & & 0.07 & [116] \\
\hline & & & & $\begin{array}{l}\text { (d) FIRAS [7] (Fig. 3), } \\
\text { with recalibration in [33] }\end{array}$ \\
\hline 33 & 2.71548 & & 0.060 & (d) \\
\hline 43 & 2.73548 & & 0.044 & (d) \\
\hline 50 & 2.73048 & & 0.033 & (d) \\
\hline 58 & 2.72548 & & 0.022 & (d) \\
\hline \multirow[t]{2}{*}{67} & 2.72548 & & 0.016 & (d) \\
\hline & & & & $\begin{array}{l}\text { (e) FIRAS [32], with } \\
\text { recalibration in [33] }\end{array}$ \\
\hline 68.1 & 2.72552 & & 0.00011 & \\
\hline 81.5 & 2.72553 & & 0.00011 & (e) \\
\hline 95.3 & 2.72555 & & 0.00011 & (e) \\
\hline 108.8 & 2.72549 & & 0.00009 & (e) \\
\hline 122.3 & 2.72554 & & 0.00007 & (e) \\
\hline 136.1 & 2.72540 & & 0.00006 & (e) \\
\hline 149.6 & 2.72540 & & 0.00005 & (e) \\
\hline 163.4 & 2.72546 & & 0.00004 & (e) \\
\hline 176.9 & 2.72555 & & 0.00004 & (e) \\
\hline 190.4 & 2.72549 & & 0.00003 & (e) \\
\hline 204.2 & 2.72548 & & 0.00003 & (e) \\
\hline 217.6 & 2.72551 & & 0.00002 & (e) \\
\hline 231.1 & 2.72543 & & 0.00002 & (e) \\
\hline 244.9 & 2.72550 & & 0.00002 & (e) \\
\hline 258.4 & 2.72550 & & 0.00002 & (e) \\
\hline 272.2 & 2.72543 & & 0.00003 & (e) \\
\hline 285.7 & 2.72550 & & 0.00003 & (e) \\
\hline 299.2 & 2.72551 & & 0.00004 & (e) \\
\hline 313.0 & 2.72551 & & 0.00005 & (e) \\
\hline 326.5 & 2.72540 & & 0.00006 & (e) \\
\hline 340.0 & 2.72534 & & 0.00007 & (e) \\
\hline 353.8 & 2.72568 & & 0.00008 & (e) \\
\hline 367.2 & 2.72550 & & 0.00008 & (e) \\
\hline 381.0 & 2.72546 & & 0.00009 & (e) \\
\hline 394.5 & 2.72551 & & 0.00010 & (e) \\
\hline 408.0 & 2.72540 & & 0.00010 & (e) \\
\hline 421.8 & 2.72551 & & 0.00011 & (e) \\
\hline 435.3 & 2.72564 & & 0.00012 & (e) \\
\hline 448.8 & 2.72540 & & 0.00013 & (e) \\
\hline
\end{tabular}


TABLE I. (Continued.)

\begin{tabular}{|c|c|c|c|c|}
\hline$v(\mathrm{GHz})$ & $\begin{array}{l}T(\mathrm{~K}) \\
\text { (a) }\end{array}$ & $\begin{array}{l}T(\mathrm{~K}) \\
\quad(\mathrm{b})\end{array}$ & $\begin{array}{l}1 \sigma \text { error }(\mathrm{K}) \\
\text { (c) }\end{array}$ & $\begin{array}{c}\text { Reference } \\
\text { From FIRAS [32], } \\
\text { with recalibration in [33] }\end{array}$ \\
\hline 462.6 & 2.72533 & & 0.00015 & (e) \\
\hline 476.1 & 2.72555 & & 0.00019 & (e) \\
\hline 489.9 & 2.72555 & & 0.00023 & (e) \\
\hline 503.4 & 2.72564 & & 0.00030 & (e) \\
\hline 516.8 & 2.72505 & & 0.00037 & (e) \\
\hline 530.6 & 2.72557 & & 0.00045 & (e) \\
\hline 544.1 & 2.72593 & & 0.00055 & (e) \\
\hline 557.9 & 2.72496 & & 0.00066 & (e) \\
\hline 571.4 & 2.72534 & & 0.00080 & (e) \\
\hline 584.9 & 2.72569 & & 0.00108 & (e) \\
\hline 598.7 & 2.72628 & & 0.00168 & (e) \\
\hline 612.2 & 2.72750 & & 0.00311 & (e) \\
\hline 625.7 & 2.72064 & & 0.00652 & (e) \\
\hline \multirow[t]{2}{*}{639.5} & 2.70382 & & 0.01468 & (e) \\
\hline & (a) & (b) & & $\begin{array}{l}\text { (f) [34], subtracting the global contribution } \\
\text { by unresolved extragalactic radio sources }\end{array}$ \\
\hline 0.04 & 4317 & 3874 & 963 & (f) \\
\hline 0.05 & 2645 & 2405 & 526 & (f) \\
\hline 0.06 & 1880 & 1735 & 365 & (f) \\
\hline 0.07 & 1189 & 1094 & 208 & (f) \\
\hline 0.08 & 969 & 903 & 112 & (f) \\
\hline
\end{tabular}

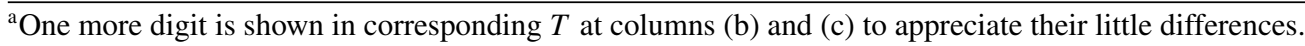

${ }^{\mathrm{b}}$ We add statistic and systematic errors in quadrature.

the (almost) complete available sets of data. Indeed, such a (relatively high) value is not surprising, since different data sets are affected by different systematic effects and derived with different foreground treatments. Moreover, we are assuming just a simple model as in (17).

For an immediate comparison (and cross-check), we will now consider a smaller data set, i.e., only the data in Table 1 of [10], but using the full set of FIRAS data and not replacing it with the condensed FIRAS value at $250 \mathrm{GHz}$. In this case we obtain a reduced $\chi^{2} \simeq 1.08$ with best-fit parameters (and again errors at $95 \%$ confidence level) $v_{0}=0.66 \pm 0.06 \mathrm{GHz}$ and $\alpha=2.55 \pm 0.10$ [implying a power-law amplitude of $18.72 \mathrm{~K}$ at $0.31 \mathrm{GHz}$, formally in terms of equivalent thermodynamic temperature; see (17)], fully consistent within errors with those found in Table 2 of [10] for the power-law fit model, as expected. In Fig. 3 we compare our best fit of the smaller data set with the best fit of the full data set which is discussed in the main text.

Applying the higher extragalactic subtraction, we find a similar reduced $\chi^{2}(\simeq 1.07), v_{0} \simeq 0.64 \pm 0.06 \mathrm{GHz}$, and, as expected, a slightly smaller value of $\alpha(\simeq 2.52 \pm 0.11)$. Finally, for both extragalactic subtraction models, replacing the full set of FIRAS data with the condensed FIRAS value at $250 \mathrm{GHz}$, we find similar best-fit values, but with a reduced $\chi^{2} \simeq 1.6$, in agreement with the one found in [10]. This simple comparison between the results found using two different data-set compilations underlines the relevance of a significant improvement of both background observations and foreground modeling.

\section{APPENDIX B: GLOBAL PHOTON ENERGY AND NUMBER DENSITY}

In this Appendix we discuss the frequency range validity for the assumed $D(v)$ or $\phi(v)$, from (11), in the modified

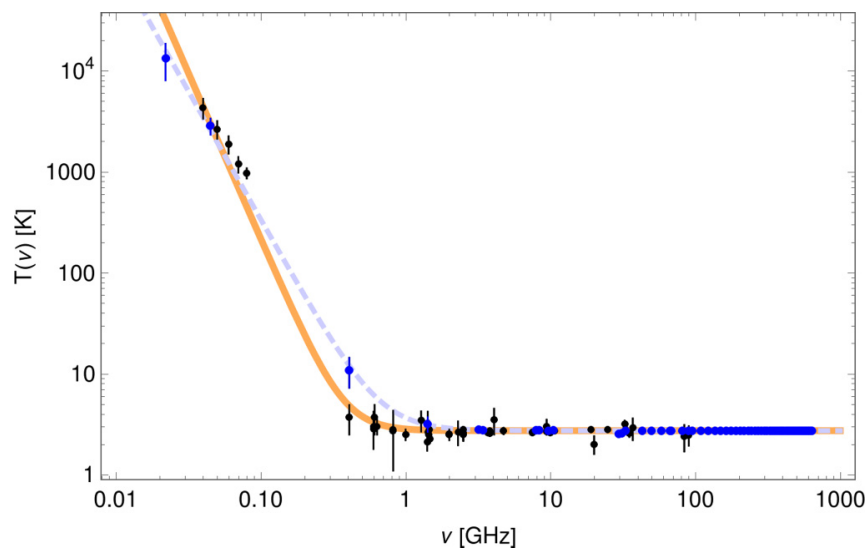

FIG. 3. Cosmic background absolute temperature as a function of the frequency (in gigahertz). The experimental data discussed in the main text (with smaller subtraction of extragalactic signal) are shown as blue and black dots with $1 \sigma$ error bars. The data subset from [10] with a full set of FIRAS data is shown as blue dots. The light blue dashed line is the best fit to the latter (see the text for the inferred parameters). For comparison we plot here also the best fit of the full data set (orange solid line), which is discussed in the main text. 
Kompaneets equation (10) [see (13)-(16) and also (3)]. We rewrite the (nonequilibrium stationary) photon occupation number $n(v)$ as

$$
\begin{aligned}
n(v) & =\frac{1}{e^{\phi(v)}-1} \\
& =n_{P}(v)+\left[n(v)-n_{P}(v)\right] \\
& =n_{P}(v)+\delta n(v),
\end{aligned}
$$

where $n_{P}(v)=1 /\left(e^{x_{e}}-1\right)$ is the Planckian distribution, $x_{e}=$ $h v / k_{\mathrm{B}} T_{e}$, and $\delta n(v)$ defines the departure of $n(v)$ from it. To calculate $\delta n(v)$ at low frequencies, where the excess is more relevant, we can rely on the Rayleigh-Jeans approximation

$$
\begin{aligned}
\delta n(v) & \simeq n(v)^{\mathrm{RJ}}-n_{P}(v)^{\mathrm{RJ}} \\
& \simeq \frac{1}{x_{e}+C\left(x_{e}\right)}-\frac{1}{x_{e}} \\
& =x_{e, 0}^{\alpha} x_{e}^{-(\alpha+1)},
\end{aligned}
$$

simplifying the computation of the global photon energy and number density:

$$
\begin{aligned}
E_{r} & =8 \pi \frac{\left(k_{\mathrm{B}} T_{e}\right)^{4}}{(h c)^{3}} \int_{0}^{\infty} n\left(x_{e}\right) x_{e}^{3} d x_{e} \\
& \simeq E_{P}+8 \pi \frac{\left(k_{\mathrm{B}} T_{e}\right)^{4}}{(h c)^{3}} \int_{x_{a}}^{x_{b}} x_{e, 0}^{\alpha} x_{e}^{-(\alpha+1)} x_{e}^{3} d x_{e}, \\
N_{r} & =8 \pi \frac{\left(k_{\mathrm{B}} T_{e}\right)^{3}}{(h c)^{3}} \int_{0}^{\infty} n\left(x_{e}\right) x_{e}^{2} d x_{e} \\
& \simeq N_{P}+8 \pi \frac{\left(k_{\mathrm{B}} T_{e}\right)^{3}}{(h c)^{3}} \int_{x_{a}}^{x_{b}} x_{e, 0}^{\alpha} x_{e}^{-(\alpha+1)} x_{e}^{2} d x_{e} .
\end{aligned}
$$

Here $E_{P}=a T_{e}^{4}$ and $N_{P}=\left(I_{2} / I_{3}\right)\left(a T_{e}^{3} / k_{\mathrm{B}}\right)$ are the global photon energy and number density for the Planckian distribution, $a=8 \pi I_{3} k_{\mathrm{B}}^{4} /(h c)^{3}, I_{m}=\int_{0}^{\infty} x^{m}\left[e^{x}-1\right]^{-1} d x=$ $m ! \zeta(m+1)\left(I_{2} \simeq 2.404\right.$ and $\left.I_{3}=\pi^{4} / 15\right), x_{e, 0}=h v_{0} / k_{\mathrm{B}} T_{e}$, and $x_{a}$ and $x_{b}$ (with $x_{a} \ll x_{e, 0} \ll 1 \lesssim x_{b}$ ) define the integration interval in $x_{e}$. Let us write

$$
E_{r} \simeq E_{P} \tilde{f}\left(x_{e, 0}, x_{a}, x_{b}, \alpha\right)
$$

and

$$
N_{r} \simeq N_{P} \tilde{\varphi}\left(x_{e, 0}, x_{a}, x_{b}, \alpha\right) .
$$

For $\alpha=3$ we get

$$
\tilde{f}\left(x_{e, 0}, x_{a}, x_{b}, \alpha\right)=1+\left(15 / \pi^{4}\right) x_{e, 0}^{\alpha} \ln \left(x_{b} / x_{a}\right),
$$

while for $\alpha \neq 3$ we have

$\tilde{f}\left(x_{e, 0}, x_{a}, x_{b}, \alpha\right)=1+\frac{15}{\pi^{4}} \frac{x_{e, 0}^{\alpha}}{\alpha-3}\left(x_{a}^{-(\alpha-3)}-x_{b}^{-(\alpha-3)}\right)$.
For $\alpha=2$ we get

$$
\tilde{\varphi}\left(x_{e, 0}, x_{a}, x_{b}, \alpha\right)=1+\left(1 / I_{2}\right) x_{e, 0}^{\alpha} \ln \left(x_{b} / x_{a}\right),
$$

while for $\alpha \neq 2$ we have

$\tilde{\varphi}\left(x_{e, 0}, x_{a}, x_{b}, \alpha\right)=1+\frac{1}{I_{2}} \frac{x_{e, 0}^{\alpha}}{\alpha-2}\left(x_{a}^{-(\alpha-2)}-x_{b}^{-(\alpha-2)}\right)$.

For $\alpha<3$ (or $\alpha<2$ ), we could in principle set $x_{a} \rightarrow 0$ in the calculation of $E_{r}$ (or of $N_{r}$ ), but the result depends also on $x_{b}$ and obviously $\delta n(v)$ could become appreciable also at relatively larger $x_{e}$ for decreasing $\alpha$, possibly requiring us to go beyond the Rayleigh-Jeans limit for a precise calculation. Conversely, for $\alpha=3$ (B7) gives $(\tilde{f}-1) \simeq$ $-\left(15 / \pi^{4}\right) x_{e, 0}^{\alpha} \ln \left(x_{a}\right)$, while for $\alpha=2$ (B10) gives $(\tilde{\varphi}-1) \simeq$ $-\left(1 / I_{2}\right) x_{e, 0}^{\alpha} \ln \left(x_{a}\right)$, implying a formal divergence for $x_{a} \rightarrow 0$. Analogously, considering that $x_{a} \ll x_{b}$, (B8) gives $(\tilde{f}-1) \simeq$ $\left(15 / \pi^{4}\right)\left[x_{e, 0}^{\alpha} /(\alpha-3)\right] x_{a}^{-(\alpha-3)}$ for $\alpha$ sufficiently larger than 3 , while (B10) gives $(\tilde{\varphi}-1) \simeq\left(1 / I_{2}\right)\left[x_{e, 0}^{\alpha} /(\alpha-2)\right] x_{a}^{-(\alpha-2)}$ for $\alpha$ sufficiently larger than 2 ; in general, $\alpha>3$ (or $\alpha>2$ ) implies again a divergence of $E_{r}$ (or of $N_{r}$ ) for $x_{a} \rightarrow 0$. More physically, $n\left(x_{e}\right)$ should have a substantial flattening at $x_{e}$ below a certain dimensionless frequency $x_{a}$ (or at $v$ below a present time frequency $v_{a}$ ).

The relative difference of the global photon energy density with respect to the Planckian case, $\delta E_{r} / E_{P}=\left(E_{r}-\right.$ $\left.E_{P}\right) / E_{P} \simeq \tilde{f}-1$, is less than a certain value $\epsilon(\ll 1)$ for $x_{a} \gtrsim$ $\exp \left[\left(15 / \pi^{4}\right) x_{e, 0}^{-3} \epsilon\right]$ if $\alpha=3$ or for $x_{a} \gtrsim\left[(15 / \pi)\left(x_{e, 0}^{\alpha} / \epsilon\right) /(\alpha-\right.$ $3)]^{1 /(\alpha-3)}$ if $\alpha>3$. Analogously, for $\alpha>2$, the relative difference of the global photon number density with respect to the Planckian case, $\delta N_{r} / N_{P}=\left(N_{r}-N_{P}\right) / N_{P} \simeq \tilde{\varphi}-1$, is less than $\epsilon$ for $x_{a} \gtrsim\left[\left(1 / I_{2}\right)\left(x_{e, 0}^{\alpha} / \epsilon\right) /(\alpha-2)\right]^{1 /(\alpha-2)}$.

The requirement of a change in the redshift of matterradiation equivalence less than $\sim 1 \%$, comparable to the accuracy set by Planck [75], i.e., $\epsilon \sim 10^{-2}$ (a condition stronger than that set by standard cosmological nucleosynthesis), in the case of the best-fit values of $v_{0}$ and $\alpha$ found in Sec. V, implies $x_{a} \gtrsim 2.3 \times 10^{-14}$, corresponding to $v_{a} \gtrsim 1.3 \times 10^{-3} \mathrm{~Hz}$, which is certainly not stringent. For comparison, a much stronger condition $\delta E_{r} / E_{P} \lesssim 10^{-5}$ (not to be confused with the potential limits on spectral distortion parameters from analyses in the near-equilibrium approach usually performed at higher frequencies) requires $x_{a} \gtrsim 2.3 \times 10^{-4}$, corresponding to $v_{a} \gtrsim$ $0.013 \mathrm{GHz}$, a value approaching the minimum frequency of current cosmic background observations. In the case $\alpha=$ 3 , for any significant value of $\epsilon$, we find instead $x_{a}$ larger than a value always negligible in practice, as expected from continuity with the case $\alpha<3$.
[1] L. Danese and G. de Zotti, The relic radiation spectrum and the thermal history of the universe, Nuovo Cim. Riv. Ser. 7, 277 (1977).

[2] R. A. Sunyaev and R. Khatri, Unavoidable CMB spectral features and blackbody photosphere of our universe, Int. J. Mod. Phys. D 22, 1330014 (2013).

[3] R. Barkana and A. Loeb, In the beginning: The first sources of light and the reionization of the universe, Phys. Rep. 349, 125 (2001).
[4] A. Lewis and A. Challinor, Weak gravitational lensing of the CMB, Phys. Rep. 429, 1 (2006).

[5] R. Adam et al. (Planck Collaboration), Planck intermediate results. XLVII. Planck constraints on reionization history, Astron. Astrophys. 596, A108 (2016).

[6] N. Aghanim et al. (Planck Collaboration), Planck 2018 results. VIII. Gravitational lensing, Astron. Astrophys. (2020), doi: 10.1051/0004-6361/201833886 
[7] J. C. Mather, E. S. Cheng, R. E. Eplee, Jr., R. B. Isaacman, S. S. Meyer, R. A. Shafer, R. Weiss, E. L. Wright, C. L. Bennett, N. W. Boggess et al., A preliminary measurement of the cosmic microwave background spectrum by the Cosmic Background Explorer (COBE) satellite, Astrophys. J. Lett. 354, L37 (1990).

[8] D. J. Fixsen, A. Kogut, S. Levin, M. Limon, P. Lubin, P. Mirel, M. Seiffert, J. Singal, E. Wollack, T. Villela, and C. A. Wuensche, ARCADE 2 measurement of the absolute sky brightness at 3-90 GHz, Astrophys. J. 734, 5 (2011).

[9] J. D. Bowman, A. E. E. Rogers, R. A. Monsalve, T. J. Mozdzen, and N. Mahesh, An absorption profile centered at 78 megahertz in the sky-averaged spectrum, Nature (London) 555, 67 (2018).

[10] M. Seiffert, D. J. Fixsen, A. Kogut, S. M. Levin, M. Limon, P. M. Lubin, P. Mirel, J. Singal, T. Villela, E. Wollack, and C. A. Wuensche, Interpretation of the ARCADE 2 absolute sky brightness measurement, Astrophys. J. 734, 6 (2011).

[11] R. Barkana, Possible interaction between baryons and darkmatter particles revealed by the first stars, Nature (London) 555, 71 (2018).

[12] A. S. Kompaneets, The establishment of thermal equilibrium between quanta and electrons, Zh. Eksp. Teor. Fiz. 31, 876 (1956) [Sov. Phys. JETP 4, 730 (1957)].

[13] S. M. Ulam, in Proceedings of the Fourth Berkeley Symposium on Mathematical Statistics and Probability, edited by J. Neyman (University of California Press, Berkeley, 1961), pp. 315-320.

[14] P. A. Sturrock, Stochastic acceleration, Phys. Rev. 141, 186 (1966).

[15] B. Aguer, S. De Bièvre, P. Lafitte, and P. E. Parris, Classical motion in force fields with short range correlations, J. Stat. Phys. 138, 780 (2010).

[16] U. Basu and C. Maes, Nonequilibrium response and frenesy, J. Phys.: Conf. Ser. 638, 012001 (2015).

[17] J. J. Hopfield, Kinetic proofreading: A new mechanism for reducing errors in biosynthetic processes requiring high specificity, Proc. Natl. Acad. Sci. USA 71, 4135 (1974).

[18] T. Demaerel, W. De Roeck, and C. Maes, Producing suprathermal tails in the stationary velocity distribution, Physica A, 122179 (2019).

[19] R. Landauer, Inadequacy of entropy and entropy derivatives in characterizing the steady state, Phys. Rev. A 12, 636 (1975).

[20] C. Maes and K. Netočný, Heat bounds and the blowtorch theorem, Ann. Henri Poinc. 4, 1193 (2013).

[21] R. R. Exartier and L. Peliti, Measuring effective temperatures in out-of-equilibrium driven systems, Eur. Phys. J. B 16, 119 (2000).

[22] L. F. Cugliandolo, J. Kurchan, and L. Peliti, Energy flow, partial equilibration, and effective temperatures in systems with slow dynamics, Phys. Rev. E 55, 3898 (1997).

[23] J. M. Ortiz de Zarate and J. V. Sengers, Hydrodynamic Fluctuations in Fluids and Fluid Mixtures (Elsevier, Amsterdam, 2006).

[24] A. Crisanti, A. Puglisi, and D. Villamaina, Nonequilibrium and information: The role of cross correlations, Phys. Rev. E 85, 061127 (2012).

[25] I. Nardecchia, J. Torres, M. Lechelon, V. Giliberti, M. Ortolani, P. Nouvel, M. Gori, Y. Meriguet, I. Donato, J. Preto, L. Varani, J. Sturgis, and M. Pettini, Out-of-Equilibrium
Collective Oscillation as Phonon Condensation in a Model Protein, Phys. Rev. X 8, 031061 (2018).

[26] L. Conti, P. De Gregorio, G. Karapetyan, C. Lazzaro, M. Pegoraro, M. Bonaldi, and L. Rondoni, Effects of breaking vibrational energy equipartition on measurements of temperature in macroscopic oscillators subject to heat flux, J. Stat. Mech. (2013) P12003.

[27] G. Falasco, M. V. Gnann, D. Rings, and K. Kroy, Effective temperatures of hot Brownian motion, Phys. Rev. E 90, 032131 (2014).

[28] G. Falasco, M. Baiesi, L. Molinaro, L. Conti, and F. Baldovin, Energy repartition for a harmonic chain with local reservoirs, Phys. Rev. E 92, 022129 (2015).

[29] A. A. Penzias and R. W. Wilson, A measurement of excess antenna temperature at $4080 \mathrm{Mc} / \mathrm{s}$, Astrophys. J. 142, 419 (1965).

[30] R. Salvaterra and C. Burigana, A joint study of early and late spectral distortions of the cosmic microwave background and of the millimetric foreground, Mon. Not. R. Astron. Soc. 336, 592 (2002).

[31] M. Gervasi, M. Zannoni, A. Tartari, G. Boella, and G. Sironi, TRIS. II. Search for CMB spectral distortions at 0.60, 0.82, and $2.5 \mathrm{GHz}$, Astrophys. J. 688, 24 (2008).

[32] D. J. Fixsen, E. S. Cheng, J. M. Gales, J. C. Mather, R. A. Shafer, and E. L. Wright, The cosmic microwave background spectrum from the full COBE FIRAS data set, Astrophys. J. 473, 576 (1996).

[33] D. J. Fixsen, The temperature of the cosmic microwave background, Astrophys. J. 707, 916 (2009).

[34] J. Dowell and G. B. Taylor, The radio background below 100 MHz, Astrophys. J. Lett. 858, L9 (2018).

[35] M. Gervasi, A. Tartari, M. Zannoni, G. Boella, and G. Sironi, The contribution of the unresolved extragalactic radio sources to the brightness temperature of the sky, Astrophys. J. 682, 223 (2008).

[36] I. Prandoni, G. Guglielmino, R. Morganti, M. Vaccari, A. Maini, H. J. A. Röttgering, M. J. Jarvis, and M. A. Garrett, The Lockman Hole Project: New constraints on the sub-mJy source counts from a wide-area $1.4 \mathrm{GHz}$ mosaic, Mon. Not. R. Astron. Soc. 481, 4548 (2018).

[37] E. Retana-Montenegro, H. J. A. Röttgering, T. W. Shimwell, R. J. van Weeren, I. Prandoni, G. Brunetti, P. N. Best, and M. Brüggen, Deep LOFAR $150 \mathrm{MHz}$ imaging of the Boötes field: Unveiling the faint low-frequency sky, Astron. Astrophys. 620, A74 (2018).

[38] T. Vernstrom, D. Scott, J. V. Wall, J. J. Condon, W. D. Cotton, E. B. Fomalont, K. I. Kellermann, N. Miller, and R. A. Perley, Deep $3 \mathrm{GHz}$ number counts from a $P(D)$ fluctuation analysis, Mon. Not. R. Astron. Soc. 440, 2791 (2014).

[39] T. Vernstrom, D. Scott, J. V. Wall, J. J. Condon, W. D. Cotton, K. I. Kellermann, and R. A. Perley, Deep 3-GHz observations of the Lockman Hole North with the Very Large Array-II. Catalogue and $\mu \mathrm{Jy}$ source properties, Mon. Not. R. Astron. Soc. 462, 2934 (2016).

[40] T. Trombetti and C. Burigana, Semi-analytical description of clumping factor and cosmic microwave background free-free distortions from reionization, Mon. Not. R. Astron. Soc. 437, 2507 (2014).

[41] S. P. Oh, Observational signatures of the first luminous objects, Astrophys. J. 527, 16 (1999). 
[42] N. Fornengo, R. Lineros, M. Regis, and M. Taoso, Possibility of a Dark Matter Interpretation for the Excess in Isotropic Radio Emission Reported by Arcade, Phys. Rev. Lett. 107, 271302 (2011).

[43] P. L. Biermann, B. B. Nath, L. I. Caramete, B. C. Harms, T. Stanev, and J. Becker Tjus, Cosmic backgrounds due to the formation of the first generation of supermassive black holes, Mon. Not. R. Astron. Soc. 441, 1147 (2014).

[44] C. Feng and G. Holder, Enhanced global signal of neutral hydrogen due to excess radiation at cosmic dawn, Astrophys. J. Lett. 858, L17 (2018).

[45] A. Ewall-Wice, T.-C. Chang, J. Lazio, O. Doré, M. Seiffert, and R. A. Monsalve, Modeling the radio background from the first black holes at cosmic dawn: Implications for the $21 \mathrm{~cm}$ absorption amplitude, Astrophys. J. 868, 63 (2018).

[46] I. F. Mirabel, Black hole high mass X-ray binary microquasars at cosmic dawn, Proc. Int. Astron. Union 14, 365 (2018).

[47] R. Subrahmanyan and R. Cowsik, Is there an unaccounted for excess in the extragalactic cosmic radio background? Astrophys. J. 776, 42 (2013).

[48] R. Hills, G. Kulkarni, P. D. Meerburg, and E. Puchwein, Concerns about modeling of the EDGES data, Nature (London) 564, E32 (2018).

[49] P. Sharma, Astrophysical radio background cannot explain the EDGES 21-cm signal: Constraints from cooling of non-thermal electrons, Mon. Not. R. Astron. Soc. 481, L6 (2018).

[50] S. Singh and R. Subrahmanyan, The redshifted $21 \mathrm{~cm}$ signal in the EDGES low-band spectrum, Astrophys. J. 880, 26 (2019).

[51] R. Weymann, Diffusion approximation for a photon gas interacting with a plasma via the Compton effect, Phys. Fluids 8, 2112 (1965).

[52] G. B. Rybicki and A. P. Lightman, Radiative Processes in Astrophysics (Wiley-Interscience, Hoboken, 1979).

[53] J. I. Katz, High Energy Astrophysics, Frontiers in Physics Lecture Note Series (Addison-Wesley, Reading, 1987).

[54] C. W. Gardiner, Handbook of Stochastic Methods for Physics, Chemistry and the Natural Sciences, 3rd ed., Springer Series in Synergetics Vol. 13 (Springer, Berlin, 2004).

[55] C. Burigana, L. Danese, and G. de Zotti, Formation and evolution of early distortions of the microwave background spectrum-A numerical study, Astron. Astrophys. 246, 49 (1991).

[56] A. P. Lightman, Double Compton emission in radiation dominated thermal plasmas, Astrophys. J. 244, 392 (1981).

[57] L. Danese and G. de Zotti, Double Compton process and the spectrum of the microwave background, Astron. Astrophys. 107, 39 (1982).

[58] R. J. Gould, The cross section for double Compton scattering, Astrophys. J. 285, 275 (1984).

[59] W. J. Karzas and R. Latter, Electron radiative transitions in a Coulomb field, Astrophys. J. Suppl. 6, 167 (1961).

[60] G. B. Rybicki and A. P. Lightman, Radiative Processes in Astrophysics (Wiley-VCH, Weinheim, 1986).

[61] A. Zizzo and C. Burigana, On the effect of cyclotron emission on the spectral distortions of the cosmic microwave background, New Astron. 11, 1 (2005).

[62] J. Chluba and R. A. Sunyaev, The evolution of CMB spectral distortions in the early universe, Mon. Not. R. Astron. Soc. 419, 1294 (2012).
[63] L. van Hove, Quantum-mechanical perturbations giving rise to a statistical transport equation, Physica 21, 517 (1955).

[64] L. Van Hove, The approach to equilibrium in quantum statistics: A perturbation treatment to general order, Physica 23, 441 (1957).

[65] E. Fermi, On the origin of the cosmic radiation, Phys. Rev. 75, 1169 (1949).

[66] G. I. Taylor, Diffusion by continuous movements, Proc. Lond. Math. Soc. 20, 196 (1922).

[67] J. Eckman and N. Cuneo, Non-equilibrium steady states for chains of four rotors, Commun. Math. Phys. 345, 185 (2016).

[68] N. Cuneo, J. Eckmann, and C. E. Wayne, Energy dissipation in Hamiltonian chains of rotators, Nonlinearity 30, R81 (2017).

[69] S. Iubini, L. Chirondojan, G.-L. Oppo, A. Politi, and P. Politi, Dynamical Freezing of Relaxation to Equilibrium, Phys. Rev. Lett. 122, 084102 (2019).

[70] G. Grimmett and D. Stirzaker, Probability and Random Processes, 2nd ed. (Clarendon Press, Oxford, 1992), Sec. 13.3.

[71] R. Penrose, The Road to Reality: A Complete Guide to the Laws of the Universe (Vintage Books, London, 2005).

[72] L. S. Brown, Compton scattering in a plasma, Ann. Phys. (NY) 200, 190 (1990).

[73] R. A. Sunyaev, Induced Compton scattering by thermal electrons and the low-frequency spectrum of radio sources, Sov. Astron. 15, 190 (1971).

[74] S. J. Tanaka, K. Asano, and T. Terasawa, Avalanche photon cooling by induced Compton scattering: Higher-order Kompaneets equation, Prog. Theor. Exp. Phys. 2015, 073 E01 (2015).

[75] N. Aghanim et al. (Planck Collaboration), Planck 2018 results. VI. Cosmological parameters, arXiv:1807.06209.

[76] R. S. Roger, C. H. Costain, T. L. Landecker, and C. M. Swerdlyk, The radio emission from the galaxy at $22 \mathrm{MHz}$, Astron. Astrophys. Suppl. Ser. 137, 7 (1999).

[77] K. Maeda, H. Alvarez, J. Aparici, J. May, and P. Reich, A 45$\mathrm{MHz}$ continuum survey of the northern hemisphere, Astron. Astrophys. Suppl. Ser. 140, 145 (1999).

[78] C. G. T. Haslam, U. Klein, C. J. Salter, H. Stoffel, W. E. Wilson, M. N. Cleary, D. J. Cooke, and P. Thomasson, A $408 \mathrm{MHz}$ all-sky continuum survey. I-Observations at southern declinations and for the north polar region, Astron. Astrophys. 100, 209 (1981).

[79] P. Reich and W. Reich, A radio continuum survey of the northern sky at $1420 \mathrm{MHz}$. II, Astron. Astrophys. Suppl. 63, 205 (1986).

[80] T. F. Howell and J. R. Shakeshaft, Spectrum of the $3^{\circ} \mathrm{K}$ cosmic microwave radiation, Nature (London) 216, 753 (1967).

[81] K. S. Stankevich, R. Wielebinski, and W. E. Wilson, Radio sky background studies using the Moon as a screen, Aust. J. Phys. 23, 529 (1970).

[82] S. A. Pelyushenko and K. S. Stankevich, Absolute temperature measurements of the cosmic radio emission of the sky in the decimeter range, Sov. Astron. 13, 223 (1969).

[83] A. A. Penzias and R. W. Wilson, A measurement of the background temperature at $1415 \mathrm{MHz}$, Astron. J. 72, 315 (1967).

[84] T. F. Howell and J. R. Shakeshaft, Measurement of the minimum cosmic background radiation at $20.7-\mathrm{cm}$ wave-length, Nature (London) 210, 1318 (1966). 
[85] T. Y. Otoshi and C. T. Stelzreid, Cosmic background noise temperature measurements at $13-\mathrm{cm}$ wavelength, IEEE Trans. Instrum. Meas. 24, 174 (1975).

[86] P. G. Roll and D. T. Wilkinson, Cosmic Background Radiation at $3.2 \mathrm{~cm}$-Support for Cosmic Black-Body Radiation, Phys. Rev. Lett. 16, 405 (1966).

[87] R. A. Stokes, R. B. Partridge, and D. T. Wilkinson, New Measurements of the Cosmic Microwave Background at $\lambda=$ $3.2 \mathrm{~cm}$ and $\lambda=1.58 \mathrm{~cm}$-Evidence in Support of a Blackbody Spectrum, Phys. Rev. Lett. 19, 1199 (1967).

[88] W. J. Welch, S. Keachie, D. D. Thornton, and G. Wrixon, Measurement of the Cosmic Microwave Background Temperature at 1.5-cm Wavelength, Phys. Rev. Lett. 18, 1068 (1967).

[89] M. S. Ewing, B. F. Burke, and D. H. Staelin, Cosmic Background Measurement at a Wavelength of $9.24 \mathrm{~mm}$, Phys. Rev. Lett. 19, 1251 (1967).

[90] D. T. Wilkinson, Measurement of the Cosmic Microwave Background at 8.56-mm Wavelength, Phys. Rev. Lett. 19, 1195 (1967).

[91] V. I. Puzanov, A. E. Salomonovich, and K. S. Stankevich, Measurements of the temperature of the primordial background radiation at 8.2-mm wavelength, Sov. Astron. 11, 905 (1968).

[92] A. G. Kislyakov, V. I. Chernyshev, Y. V. Lebskii, V. A. Mal'Tsev, and N. V. Serov, Effective temperature of cosmic blackbody radiation at $3.58 \mathrm{~mm}$, Sov. Astron. 15, 29 (1971).

[93] P. E. Boynton, R. A. Stokes, and D. T. Wilkinson, Primeval Fireball Intensity at $\lambda=3.3 \mathrm{~mm}$, Phys. Rev. Lett. 21, 462 (1968).

[94] M. F. Millea, M. McColl, R. J. Pedersen, and F. L. Vernon, Cosmic Background Radiation at $\lambda=3.3 \mathrm{~mm}$, Phys. Rev. Lett. 26, 919 (1971).

[95] P. E. Boynton and R. A. Stokes, Airborne measurement of the temperature of the cosmic microwave background at $3.3 \mathrm{~mm}$, Nature (London) 247, 528 (1974).

[96] G. Sironi, M. Limon, G. Marcellino, G. Bonelli, M. Bersanelli, G. Conti, and K. Reif, The absolute temperature of the sky and the temperature of the cosmic background radiation at $600 \mathrm{MHz}$, Astrophys. J. 357, 301 (1990).

[97] G. Sironi, G. Bonelli, and M. Limon, The brightness temperature of the south celestial pole and the temperature of the cosmic background radiation measured at 36.6 and 12 centimeter wavelength, Astrophys. J. 378, 550 (1991).

[98] A. Raghunathan and R. Subrahmanyan, A measurement of the cosmic microwave background temperature $1280 \mathrm{MHz}$, J. Astrophys. Astron. 21, 1 (2000).

[99] S. M. Levin, C. Witebsky, M. Bensadoun, M. Bersanelli, G. de Amici, A. Kogut, and G. F. Smoot, A measurement of the cosmic microwave background radiation temperature at 1.410 GHz, Astrophys. J. 334, 14 (1988).

[100] S. T. Staggs, N. C. Jarosik, D. T. Wilkinson, and E. J. Wollack, An absolute measurement of the cosmic microwave background radiation temperature at 20 centimeters, Astrophys. J. 458, 407 (1996).

[101] M. Bensadoun, M. Bersanelli, G. de Amici, A. Kogut, S. M. Levin, M. Limon, G. F. Smoot, and C. Witebsky,
Measurements of the cosmic microwave background temperature at $1.47 \mathrm{GHz}$, Astrophys. J. 409, 1 (1993).

[102] M. Bersanelli, M. Bensadoun, G. de Amici, S. Levin, M. Limon, and G. F. Smoot, Absolute measurement of the cosmic microwave background at $2 \mathrm{GHz}$, Astrophys. J. 424, 517 (1994).

[103] G. de Amici, M. Bersanelli, A. Kogut, S. Levin, M. Limon, and G. F. Smoot, The temperature of the cosmic microwave background radiation at $3.8 \mathrm{GHz}$ : Results of a measurement from the south pole site, Astrophys. J. 381, 341 (1991).

[104] N. Mandolesi, P. Calzolari, S. Cortiglioni, G. Morigi, L. Danese, and G. de Zotti, Measurements of the cosmic background radiation temperature at 6.3 centimeters, Astrophys. J. 310, 561 (1986).

[105] A. Kogut, M. Bensadoun, G. de Amici, S. Levin, G. F. Smoot, and C. Witebsky, A measurement of the temperature of the cosmic microwave background at a frequency of $7.5 \mathrm{GHz}$, Astrophys. J. 355, 102 (1990).

[106] S. Levin, M. Bensadoun, M. Bersanelli, G. de Amici, A. Kogut, M. Limon, and G. Smoot, A measurement of the cosmic microwave background temperature at $7.5 \mathrm{GHz}$, Astrophys. J. 396, 3 (1992).

[107] A. Kogut, M. Bersanelli, G. de Amici, S. D. Friedman, M. Griffith, B. Grossan, S. Levin, G. F. Smoot, and C. Witebsky, The temperature of the cosmic microwave background radiation at a frequency of $10 \mathrm{GHz}$, Astrophys. J. 325, 1 (1988).

[108] D. G. Johnson and D. T. Wilkinson, A $1 \%$ measurement of the temperature of the cosmic microwave radiation at lambda $=$ 1.2 centimeters, Astrophys. J. 313, L1 (1987).

[109] G. de Amici, G. Smoot, S. D. Friedman, and C. Witebsky, New $33 \mathrm{GHz}$ measurements of the cosmic background radiation intensity, Astrophys. J. 298, 710 (1985).

[110] M. Bersanelli, C. Witebsky, M. Bensadoun, G. de Amici, A. Kogut, S. M. Levin, and G. F. Smoot, Measurements of the cosmic microwave background radiation temperature at $90 \mathrm{GHz}$, Astrophys. J. 339, 632 (1989).

[111] J. A. Schuster, Ph.D. thesis, UC Berkeley, 1993.

[112] G. de Amici, G. F. Smoot, J. Aymon, M. Bersanelli, A. Kogut, S. M. Levin, and C. Witebsky, Measurement of the intensity of the cosmic background radiation at $3.7 \mathrm{GHz}$, Astrophys. J. 329, 556 (1988).

[113] N. Mandolesi, P. Calzolari, S. Cortiglioni, and G. Morigi, Measurement of the cosmic background radiation temperature at $6.3 \mathrm{~cm}$, Phys. Rev. D 29, 2680 (1984).

[114] G. Sironi, P. Inzani, and A. Ferrari, Measurement of the cosmic background radiation at $12 \mathrm{~cm}$, Phys. Rev. D 29, 2686 (1984)

[115] G. Sironi and G. Bonelli, The temperature of the diffuse background radiation at 12 centimeter wavelength, Astrophys. J. 311, 418 (1986).

[116] G. de Amici, M. Bensadoun, M. Bersanelli, A. Kogut, S. Levin, G. F. Smoot, and C. Witebsky, The temperature of the cosmic background radiation: Results from the 1987 and 1988 measurements at $3.8 \mathrm{GHz}$, Astrophys. J. 359, 219 (1990). 